\title{
Consumo de frutas y verduras por escolares adolescentes de la ciudad de Chillán. Chile.
}

\author{
Fruit and vegetable intake by adolescents \\ of Chillán City, Chile
}

\begin{abstract}
The objective of this study was to estimate adolescent fruit and vegetable intake. An analytical cross-sectional study was conducted with a random sample of 100 adolescents between the ages of 14 and 17 from a private subsidized school in Chillán. Nutritional status was evaluated according to BMI, and a food survey was applied. The survey was specially designed to capture fruit and vegetable intake and was applied by personnel with standardized training. Results: Median fruit and vegetable intake was $217 g$ (p25-p75: 139g-328g) and 183 g (p25-p75: 139g-299g) respectively; vegetable intake was higher for girls $(217 \mathrm{~g} \mathrm{v/s} 165 \mathrm{~g}$, $p=0.001$ ). Highly inadequate fruit and vegetable intake was $12 \%$ for girls and $88 \%$ for boys. Conclusions: Adolescent fruit and vegetable intake was lower than the recommended intake.

Key words: adolescents; fruit; vegetables, BMI.
\end{abstract}

Jacqueline Araneda F. (1) Marcia Ruiz N. (2)

Teresita Vallejos V. (2)

Patricio Oliva M. (1)
Este trabajo fue recibido el 16 de Abril de 2015 y aceptado para ser publicado el 25 de Julio de 2015.

consumo diaria de FV es de 168 y 230 g respectivamente, cifras que descienden a $150 \mathrm{~g}$ de frutas y $193 \mathrm{~g}$ de verduras en el grupo de 14 a 18 años. Además, destaca que los mayores consumos se registran en la Región Metropolitana y en la zona centro norte del país, como en los niveles socioeconómicos más altos (14).

Como una forma afrontar esta baja ingesta, el Ministerio de Salud de Chile, a través de las metas sanitarias 2011-2020, en el objetivo 3 relacionado con desarrollar hábitos y estilos de vida saludables que favorezcan la reducción de los factores de riesgo asociados a la carga de enfermedad de la población, ha propuesto aumentar a $20 \%$ la prevalencia de al menos 5 factores protectores, entre ellos el consumir al menos 5 porciones de FV diariamente (15).

El objetivo de la presente investigación fue estimar el consumo de FV en adolescentes que asisten a un Colegio Particular Subvencionado de la ciudad de Chillán, Región del Biobío.

\section{SUJETOS Y METODO}

Se desarrolló un estudio analítico de corte transversal. La población correspondió a estudiantes que cursan primero y segundo de enseñanza media en un Colegio Particular Subvencionado de la ciudad de Chillán. Mediante un muestreo aleatorio simple se obtuvo una muestra de 100 sujetos. El tamaño muestral se calculó con un nivel del confianza del 95\% y aceptando un error máximo permisible del 5\%.

El estudio fue aprobado por el Comité de Bioética y Bioseguridad de la Universidad del Bío-Bío. 
Ingesta Alimentaria: Previo consentimiento informado, los escolares contestaron un cuestionario de frecuencia de consumo cuantificado, validado por expertos y adolescentes del mismo colegio y grupo etáreo en estudio. Dicho cuestionario, fue realizado cara a cara con el entrevistado y por personal entrenado, indagó sobre la ingesta de FV durante el mes anterior a la entrevista, especificando los días de consumo en el mes y en la semana, así como las unidades y cantidades ingeridas. Se trabajó con un atlas alimentario que incluía un ítem específico de fotografías de FV en porciones de consumo, unidades y preparaciones. Se consideró como FV los productos que se especifican en el cuadro 1, que fueron seleccionados como los 10 más consumidos por los adolescentes encuestados, eliminando del análisis los que presentaron proporciones de consumo diario inferiores al 5\%.

Se estimó el consumo diario de FV, dividiendo la frecuencia de ingesta mensual o semanal reportada por los escolares adolescentes entre treinta o siete días respectivamente. Además los adolescentes fueron clasificados en tres categorías en relación a la ingesta diaria de FV como: a) Ingesta adecuada: si el consumo era de 400 g y más; b) Insuficiente con consumos entre 201-399 g; y c) Altamente insuficiente con consumos menores a $200 \mathrm{~g}$.

Estado nutricional: Se calculó IMC, previa medición de altura y peso por personal capacitado, utilizando técnica estandarizada $(16,17)$. El peso se midió empleando una balanza SECA modelo 714 con precisión de $100 \mathrm{~g}$ y la altura se midió con un tallímetro marca SECA modelo 714 con precisión de 0,1 milímetros. Luego, se categorizó según desviaciones estándar de acuerdo a la edad de cada adolescente medida en años y meses, con las tablas de Índice de Masa Corporal $(\mathrm{Kg} / \mathrm{m} 2)$ por edad de Niñas y Niños 5 - 19 años.

Actividad física: Se determinó mediante el reporte de los adolescentes al consultarles sobre: i) Horas diarias acostado; ii) Horas diarias de actividades frente a una pantalla (computadora, televisión, tablet o celular); iii) Horas diarias destinadas a juegos recreativos o después de la jornada escolar, iv) Horas diarias destinadas a actividades deportivas sistemáticas (talleres y/o escuelas deportivas, educación física). La actividad física de los escolares fue clasificada en tres categorías: leve, moderada e intensa.

Se calcularon las frecuencias absolutas y los porcentajes para variables cualitativas. En el caso de las variables cuantitativas se usaron medidas de tendencia central y medidas de dispersión. Se compararon las variables sexo actividad física y estado nutricional, con los niveles de consumo de FV consumidas diariamente a través de las pruebas de Kruskal Wallis y Mann-Whitney y para las variables categóricas se utilizó Chi cuadrado. El procesamiento y análisis de los datos se realizó con el software Stata, versión 11.0 (18). El nivel de confianza fue de $95 \%$, $(p<0,05)$.

\section{RESULTADOS}

La muestra estuvo constituida por 100 escolares, con representatividad similar de hombres y mujeres. La edad de los adolescentes fluctuó entre 14 y 17 años, constituyendo 50\% aquellos de 15 años. En el estado nutricional, la normalidad alcanzó la mayor proporción (50\%), seguida por el sobrepeso y obesidad con $40 \%$. Predominó la actividad física leve y moderada con proporciones cercana al 45\%, mientras que sólo $11 \%$ de los adolescentes practicaban actividad física intensa (cuadro 2).

En el cuadro 3, se observa que los adolescentes con estado nutricional de bajo peso y normalidad son los que consumen en más alto porcentaje todas las frutas, a excepción de la frambuesa que es preferida por los obesos. La totalidad de los adolescentes obesos consumen naranja y manzana diariamente. El plátano en todos los estados nutricionales tiene una proporción de consumo superior al 70\%, a excepción de los adolescentes obesos donde sólo 5 de cada 10 lo consumían. Los adolescentes con obesidad presentaban una proporción de consumo de pera, frutilla y arándanos inferior al $20 \%$.

La lechuga es la verdura más consumida en todos los estados nutricionales con proporciones de consumo superiores al $90 \%$, seguida por el tomate y la zanahoria. En el tomate la proporción disminuye a medida que aumenta la malnutrición por exceso. Los obesos consumían en menor proporción el brócoli y el pepino con cifras que no excedían el $20 \%$.

En el cuadro 4, se observa que el consumo diario de FV es mayor en mujeres, con medianas de consumo superior a los 215 g. El consumo de verduras es significativamente mayor en mujeres que en hombres (217 g v/s $165 \mathrm{~g}$ ) con diferencias que superan los 50 g diarios $(p=0,001)$.

Los escolares con estado nutricional de obesidad son los que consumen mayor cantidad de frutas con medianas superiores a 320 g diarios, mientras que los con sobrepeso consumen 170 g. En las verduras, los mayores consumos se observan en los enflaquecidos y los menores consumo en los obesos, con diferencias que alcanzan los $75 \mathrm{~g}$ (200 g v/s $120 \mathrm{~g}$ respectivamente).

Los escolares que refieren tener actividad física intensa, son los que consumen mayores cantidades de FV, con medianas de consumo superiores a 270 g en cada categoría.

El 52\% de los adolescentes cumple con la recomendación de ingesta de FV, especialmente las mujeres $(53,9 \mathrm{v} / \mathrm{s} 46,1)$,

CUADRO 1

Frutas y verduras

\begin{tabular}{lll}
\hline Frutas & Verduras \\
\hline Arándano & Apio \\
Cereza & Brócoli & Cebolla \\
Frambuesa & Coliflor \\
Frutilla & Lechuga \\
Kiwi & Pepino \\
Mandarina & Repollo \\
Naranja & Tomate \\
Pera & Zanahoria \\
Plátano & Zapallo \\
\hline
\end{tabular}


mientras que en la categoría inadecuada (entre 201 g y 399 g) es proporcionalmente igual por sexo (50\%). Los con estado nutricional enflaquecido y normal, son los que en mayor porcentaje cumplen la recomendación $(69,2 \%)$ (gráfico 1).

\section{DISCUSIÓN}

El presente estudio documenta una baja ingesta de FV en los adolescentes estudiados, en relación a las recomendaciones de la OMS, más concretamente, sólo 50\% cumple con este parámetro, destacándose un mayor consumo en mujeres que en hombres, especialmente en la ingesta de verduras.

Los resultados muestran que las frutas con mayor proporción de consumo son el plátano, manzana y naranja con cifras superiores al $70 \%$, mientras que las menos consumidas son las frutas rojas (cereza, frambuesa, frutilla) que no superan 30\%. Dentro del grupo de las verduras, el alimento con mayor proporción de consumo es la lechuga, con 95\%, seguida por el tomate y zanahoria ( $80 \%$ y $70 \%$ respectivamente). Estos resultados, coinciden con los reportados en la ENCA 2010-2012, donde destacan como las FV más consumidas: el plátano, manzana, naranja, tomate, lechuga y zanahoria (14). También, la VII Encuesta de Presupuestos Familiares desarrollada en Chile por el Instituto Nacional de Estadística (INE), reporta que una mayor proporción del gasto promedio mensual del hogar en FV, está destinado a la compra

\section{CUADRO 2}

Distribución de la muestra por sexo, edad, estado nutricional y actividad física de escolares adolescentes de Chillán.

\begin{tabular}{|c|c|c|}
\hline \multicolumn{3}{|c|}{ Muestra } \\
\hline & $\mathrm{n}$ & $\%$ \\
\hline \multicolumn{3}{|l|}{ Edad } \\
\hline 14 años & 21 & 21 \\
\hline 15 años & 50 & 50 \\
\hline 16 años & 26 & 26 \\
\hline 17 años & 3 & 3 \\
\hline \multicolumn{3}{|l|}{ Sexo } \\
\hline Femenino & 49 & 49 \\
\hline Masculino & 51 & 51 \\
\hline \multicolumn{3}{|c|}{ Estado nutricional } \\
\hline Bajo peso & 7 & 7 \\
\hline Normal & 53 & 53 \\
\hline Sobrepeso & 34 & 34 \\
\hline Obeso & 6 & 6 \\
\hline \multicolumn{3}{|c|}{ Actividad física } \\
\hline Leve & 46 & 46 \\
\hline Moderada & 43 & 43 \\
\hline Intensa & 11 & 11 \\
\hline
\end{tabular}

\section{GRAFICO 1}

Distribución porcentual de acuerdo a la recomendación de frutas y verduras.

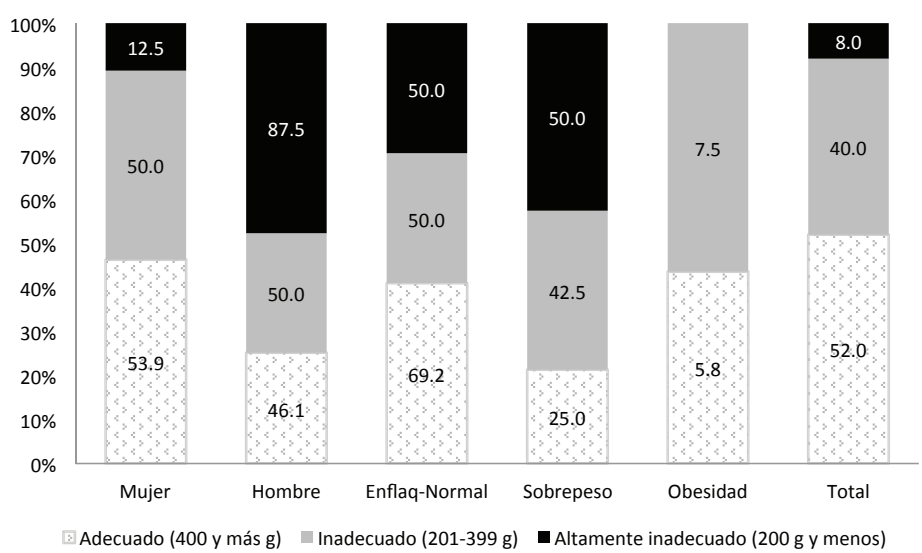


de plátano, manzana y naranja en el ítem de frutas, y al tomate en el ítem de verduras (19).

La ingesta de frutas encontrada en la presente investigación es superior a la reportada por la ENCA 2010-2012 (217 g v/s 151 g), diferencias que puede presentarse, ya que nuestra investigación sólo estudió adolescentes que asisten a un Colegio Particular Subvencionado, con alumnos matriculados principalmente de niveles socioeconómicos (NSE) altos y medios que habitan principalmente en zonas urbanas.

La ingesta de verduras de los adolescentes es similar a la reportada en la ENCA 2010-2012, con diferencias de sólo $8 \mathrm{~g}$ diarios, siendo mayor en mujeres que en hombres. Estas diferencias por sexo en el consumo de FV, también han sido documentadas en otras investigaciones. Un estudio realizado con información de 33 países europeos y norteamericanos, con un tamaño muestral de 488.951 adolescentes con edades de 11, 13 y 15 años, reportó que en todos los países estudiados las mujeres tenían más probabilidades de consumir FV que los hombres (20).

Si bien el presente trabajo encontró un bajo cumplimiento de las recomendaciones de consumo de FV, ya que sólo 50\% cumplía con los $400 \mathrm{~g}$ diarios que establece la OMS, es una proporción superior a lo encontrado en otras investigaciones. Estudios realizados en América del Norte y Europa, reportan que la mayoría de los niños y adolescentes no alcanzan la recomendación diaria

\section{CUADRO 3}

Proporción de consumo diario de frutas y verduras, según estado nutricional de escolares adolescentes de la comuna de Chillán.

\begin{tabular}{|c|c|c|c|c|}
\hline $\begin{array}{l}\text { Frutas y verduras } \\
\text { (IC inf-sup) }\end{array}$ & $\begin{array}{c}\text { Total } \\
\text { (IC inf-sup) }\end{array}$ & $\begin{array}{c}\text { Bajo peso -normal } \\
\text { (IC inf-sup) }\end{array}$ & $\begin{array}{l}\text { Sobrepeso } \\
\text { (IC inf-sup) }\end{array}$ & Obesidad \\
\hline \multicolumn{5}{|l|}{ Frutas } \\
\hline Arándanos & $\begin{array}{c}27,0 \\
(18,1-35,8)\end{array}$ & $\begin{array}{c}28,3 \\
(16,6-39,9)\end{array}$ & $\begin{array}{c}26,4 \\
(11,2-41,7)\end{array}$ & $\begin{array}{c}16,6 \\
(-16,4-49,7)\end{array}$ \\
\hline Cereza & $\begin{array}{c}23,0 \\
(14,6-31,3)\end{array}$ & $\begin{array}{c}30,0 \\
(18,1-41,8)\end{array}$ & $\begin{array}{c}11,7 \\
(0,6-22,8)\end{array}$ & $\begin{array}{c}16,7 \\
(-18,4-49,3)\end{array}$ \\
\hline Frambuesa & $\begin{array}{c}30,0 \\
(20,8-39,1)\end{array}$ & $\begin{array}{c}35,0 \\
(22,6-47,3)\end{array}$ & $\begin{array}{c}17,6 \\
(4,4-30,8)\end{array}$ & $\begin{array}{c}50,0 \\
(5,6-94,3)\end{array}$ \\
\hline Frutilla & $\begin{array}{c}32,0 \\
(22,6-41,3)\end{array}$ & $\begin{array}{c}40,0 \\
(27,3-52,6)\end{array}$ & $\begin{array}{c}20,5 \\
(6,6-34,5)\end{array}$ & $\begin{array}{c}16,6 \\
(-16,4-49,7)\end{array}$ \\
\hline Kiwi & $\begin{array}{c}26,0 \\
(17,2-34,7)\end{array}$ & $\begin{array}{c}33,3 \\
(21,1-45,5)\end{array}$ & $\begin{array}{c}17,6 \\
(4,4-30,8)\end{array}$ & 0 \\
\hline Mandarina & $\begin{array}{c}54,0 \\
(44,0-63,9)\end{array}$ & $\begin{array}{c}60,0 \\
(47,3-72,6)\end{array}$ & $\begin{array}{c}44,1 \\
(26,9-61,2)\end{array}$ & $\begin{array}{c}50,0 \\
(05,6-94,3)\end{array}$ \\
\hline Manzana & $\begin{array}{c}76,0 \\
(67,4-84,5)\end{array}$ & $\begin{array}{c}81,6 \\
(71,6-91,6)\end{array}$ & $\begin{array}{c}61,7 \\
(44,9-78,5)\end{array}$ & 100 \\
\hline Naranja & $\begin{array}{c}71,0 \\
(61,9-80,0)\end{array}$ & $\begin{array}{c}73,3 \\
(61,9-84,7)\end{array}$ & $\begin{array}{c}61,7 \\
(44,9-78,5)\end{array}$ & 100 \\
\hline Pera & $\begin{array}{c}34,0 \\
(24,5-43,4)\end{array}$ & $\begin{array}{c}36,6 \\
(24,2-49,1)\end{array}$ & $\begin{array}{c}32,3 \\
(16,1-48,5)\end{array}$ & $\begin{array}{c}16,6 \\
(-15,4-49,7)\end{array}$ \\
\hline Plátano & $\begin{array}{c}75,0 \\
(66,3-83,6)\end{array}$ & $\begin{array}{c}76,6 \\
(65,7-87,5)\end{array}$ & $\begin{array}{c}76,4 \\
(61,8-91,1)\end{array}$ & $\begin{array}{c}50,0 \\
(5,6-94,3)\end{array}$ \\
\hline \multicolumn{5}{|l|}{ Verduras } \\
\hline Apio & $\begin{array}{c}67,6 \\
(58,3-77,0)\end{array}$ & $\begin{array}{c}68,3 \\
(56,3-80,3)\end{array}$ & $\begin{array}{c}64,7 \\
(48,19-81,2)\end{array}$ & $\begin{array}{c}66,6 \\
(24,8-108,4)\end{array}$ \\
\hline Brócoli & $\begin{array}{c}49,4 \\
(39,4-59,5)\end{array}$ & $\begin{array}{c}50,0 \\
(37,0-62,9)\end{array}$ & $\begin{array}{c}55,8 \\
(38,73-73,0)\end{array}$ & $\begin{array}{c}16,6 \\
(-16,4-49,7)\end{array}$ \\
\hline Cebolla & $\begin{array}{c}58,5 \\
(48,7-68,4)\end{array}$ & $\begin{array}{c}52,5 \\
(39,5-65,5)\end{array}$ & $\begin{array}{c}67,6 \\
(51,48-83,8)\end{array}$ & $\begin{array}{c}66,6 \\
(24,8-108,5)\end{array}$ \\
\hline Coliflor & $\begin{array}{c}35,5 \\
(25,7-44,9)\end{array}$ & $\begin{array}{c}33,3 \\
(21,1-45,5)\end{array}$ & $\begin{array}{c}47,0 \\
(29,81-64,2)\end{array}$ & 0 \\
\hline Lechuga & $\begin{array}{c}94,9 \\
(90,5-99,3)\end{array}$ & $\begin{array}{c}95,0 \\
(89,3-100,6)\end{array}$ & $\begin{array}{c}94,1 \\
(85,9-102,2)\end{array}$ & 100 \\
\hline Pepino & $\begin{array}{c}35,3 \\
(25,7-44,9)\end{array}$ & $\begin{array}{c}36,6 \\
(24,2-49,1)\end{array}$ & $\begin{array}{c}35,2 \\
(18,7-51,8)\end{array}$ & $\begin{array}{c}16,6 \\
(-16,4-49,7)\end{array}$ \\
\hline Repollo & $\begin{array}{c}60,6 \\
(50,8-70,4)\end{array}$ & $\begin{array}{c}68,3 \\
(56,3-80,3)\end{array}$ & $\begin{array}{c}47,0 \\
(29,8-64,2)\end{array}$ & $\begin{array}{c}50,0 \\
(5,6-94,3)\end{array}$ \\
\hline Tomate & $\begin{array}{c}80,8 \\
(72,9-88,7)\end{array}$ & $\begin{array}{c}83,3 \\
(73,7-92,9)\end{array}$ & $\begin{array}{c}79,4 \\
(65,4-93,3)\end{array}$ & $\begin{array}{c}66,6 \\
(24,8-106,4)\end{array}$ \\
\hline Zanahoria & $\begin{array}{c}69,6 \\
(60,4-78,9)\end{array}$ & $\begin{array}{c}71,6 \\
(60,0-83,3)\end{array}$ & $\begin{array}{c}61,7 \\
(44,9-78,5)\end{array}$ & $\begin{array}{c}83,3 \\
(50,2-116,4)\end{array}$ \\
\hline Zapallo & $\begin{array}{c}46,4 \\
(36,4-56,4)\end{array}$ & $\begin{array}{c}51,6 \\
(38,7-64,5)\end{array}$ & $\begin{array}{c}38,2 \\
(21,4-55,0)\end{array}$ & $\begin{array}{c}50,0 \\
(5,6-94,4)\end{array}$ \\
\hline
\end{tabular}

Proporción e Intervalo de Confianza del 95\% 
de estos alimentos, inclusive algunos destacan que este grupo no consume estos alimentos hasta por dos días seguidos $(21,22)$. En América Latina el panorama es similar, México reporta en base a la Encuesta Nacional de Salud y Nutrición 2012 (ENSANUT 2012), que sólo 34\% de los escolares de 6 a 13 años de edad cumplía con las recomendaciones (23), y Colombia en la Encuesta Nacional de la Situación de Salud 2005 (ENSIN 2010), señala que sólo 18\% consume frutas 3 a 4 veces por semana, mientras que $16 \%$ consumía verduras diariamente (24).

En Chile, existen pocas investigaciones que indaguen sobre el consumo de FV en adolescentes, sin embargo, Olivares, en un estudio realizado en 562 niñas de niveles socioeconómicos medio- alto y bajo de edades entre los 8 y 13 años, encontró que las niñas consumen diariamente la mitad de lo recomendado, es decir $200 \mathrm{~g}$ de FV (25).

El bajo consumo de FV, anteriormente precisado puede estar relacionado con los determinantes en el consumo, expuestos por diversos autores tales como: i) falta de políticas que incentiven el consumo a nivel nacional, ii) el alto costo de las FV, iii) la baja disponibilidad y accesibilidad de estos productos en el hogar y en la escuela, iv) las preferencias y costumbres de las poblaciones, v) falta de conocimientos de los padres sobre los beneficios de la ingesta diaria de FV, vi) el mayor tiempo destinado a las preparaciones que contienen FV, vii) la baja saciedad que estos alimentos producen en relación a los procesados altos en azúcar y grasas saturadas, entre otros (26-28).

Una de las limitaciones del presente estudio es que los datos fueron obtenidos en temporada otoño-invierno, época del año que se caracteriza por una menor producción de FV en la zona y un menor consumo de éstas, en comparación con la temporada primavera-verano.

Además, en los resultados pudo influir el sesgo de memoria, ya que los adolescentes debieron recordar las FV consumidas durante un tiempo en el pasado. Por otro lado, se ha documentado que las personas reportan consumos mayores a los reales de alimentos considerados saludables como son las FV (29).

A partir del presente estudio, se abre la posibilidad de indagar a través de estudios epidemiológicos de seguimiento y con un mayor tamaño muestal, el consumo de FV y sus implicancias en la salud de los escolares. Paralelamente, se sugiere implementar estrategias en las escuelas y en el hogar, que tiendan a aumentar el consumo de FV.

Agradecimientos: A la Universidad del Bío-Bío, Chile (Proyecto Grupo de Investigación Epidemiología Alimentaria Nutricional- EPIAN- GI 131920GI/EF)

\section{RESUMEN}

El objetivo de este estudio fue estimar el consumo de frutas y verduras en adolescentes que asisten a un colegio particular subvencionado de la comuna de Chillán. Se realizó

\section{CUADRO 4}

Medianas de consumo diario de frutas y verduras, según sexo y actividad física de escolares de Chillán, Chile.

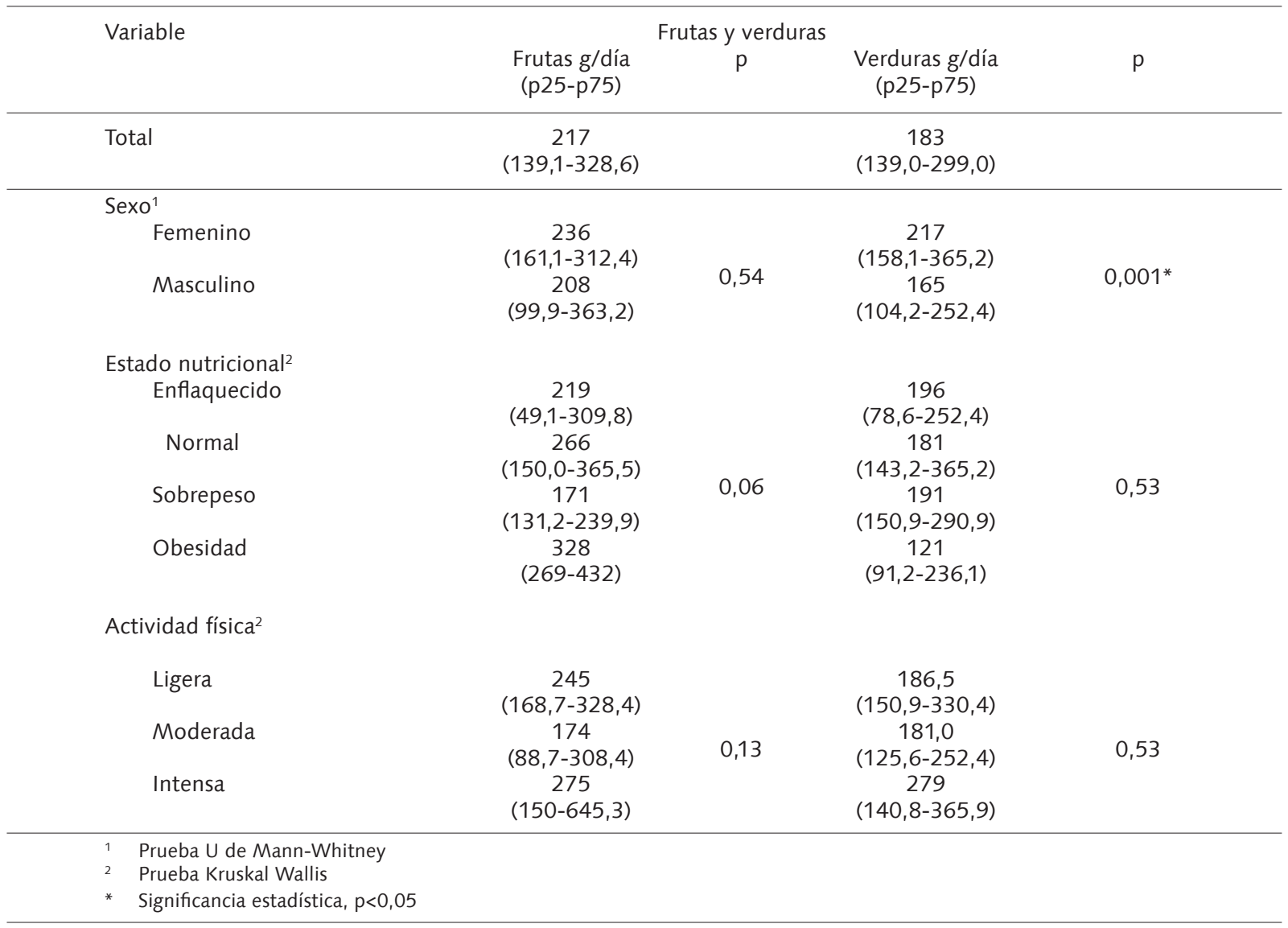


un estudio transversal analítico, con una muestra aleatoria de 100 adolescentes con edades entre los 14 y 17 años. Se evaluó estado nutricional según el IMC, y se aplicó una encuesta alimentaria, especialmente diseñada para captar el consumo de frutas y verduras, la que fue aplicada por personal capacitado y estandarizado. Resultados: La mediana de consumo de frutas y verduras fue $217 \mathrm{~g}(\mathrm{p} 25-\mathrm{p} 75=139,1-328,6)$ y 183 g (p25-p75=139,0-299,0) respectivamente, siendo mayor la ingesta de verduras en mujeres ( $217 \mathrm{v} / \mathrm{s}$ 165; $\mathrm{p}=0,001)$. Los consumos altamente inadecuados de frutas y verduras representan el $12 \%$ en mujeres y $88 \%$ en hombres. Conclusión: Los adolescentes presentan consumos de frutas y verduras bajo las recomendaciones.

Palabras clave: adolescentes; frutas; verduras, IMC.

\section{BIBLIOGRAFIA}

1. Pinto FJA. Carbajal AA. La dieta equilibrada, prudente $O$ saludable. Nutrición y salud. Instituto de Salud Pública. Madrid, 2003, p. 3-80.

2. Popkin BM, Gordon P. The nutrition transition: worldwide obesity dynamics and their determinants. Int J Obes. 2004;28:S2-S9

3. Araneda J, Bustos $P$, Cerecera $F$, Amigo $H$. Ingesta de bebidas azucaradas analcohólicas e índice de masa corporal en escolares chilenos. Salud Pública Méx. 2015; Vol. 57(2):128-34.

4. Holt E M, Steffen LM, Moran A, Basu S, Steinberger J, Ross J A, Hong Ch and Sinaiko AR. Fruit and vegetable consumption and its relation to markers of inflammation and oxidative stress in adolescents, J Am Diet Assoc. 2009; 109(3): 414-21.

5. Reiss R, Johnston J, Tucker K, DeSesso JM, Keen C. EStimation of cancer risks and benefits associated with a potential increased consumption of fruits and vegetables, Food Chem Toxicol. 2012; 50:4421-7.

6. Li M, Fan Y, Zhang X, Hou W, Tang Z. Fruit and vegetable intake and risk of type 2 diabetes mellitus: meta-analysis of prospective cohort studies. BMJ Open. 2014; 4(11):1-9.

7. Crowe FL, Roddam AW, Key TJ, et al. Fruit and vegetable intake and mortality from ischaemic heart disease: results from the European Prospective Investigation into Cancer and Nutrition (EPIC)-Heart study. Euro Heart J. 2011;32:1235-43.

8. Oyebode O, Gordon-Dseagu V, Walker A, Mindell JS. Fruit and vegetable consumption and all-cause, cancer and CVD mortality: analysis of Health Survey for England data. J Epidemiol Community Health 2014;68 (9):856-62.

9. Lampe W. Health effects of vegetables and fruit: assessing mechanisms of action in human experimental studies. Am J Clin Nutr. 1999; 70 (3) 475-90.

10. Palomo l. et al. El consumo de frutas y hortalizas ayuda a prevenir el daño endotelial. Rev Chil Nutr. 2011, 38 (3) 343-55.

11. World Health Organization/Food and Agriculture Organization. Dieta, nutrición y prevención de enfermedades crónicas. Technical series 916. Geneva: WHO/FAO, 3003.

12. GGidding SS, Dennison BA, Brich LL, Daniels SR, Gillman $M W$, Lichtenstein AH, et al. American Heart Association. Dietary recommendations for children and adolescents: a guide for practitioners. Pediatrics 2006; 117:544-59.

13. Encuesta Nacional de Salud 2009-2010. Chile. [citado 27/03/2015]. Disponible en: http://web.minsal.cl/portal/ url/item/bcb03d7bc28b64dfe040010165012d23.pdf
14. Encuesta Nacional de Consumo Alimentario (ENCA). [citado 01/03/2015]. Disponible en:http://web.minsal.cl/ sites/default/files/ENCAINFORME_FINAL.pdf

15. Gobierno de Chile. Ministerio de Salud. Estrategia nacional de salud para el cumplimiento de los objetivos sanitarios de la década 2011-2020. [citado 09/04/2015]. Disponible en: http://web.minsal.cl/portal/url/item/ c4034eddbc96ca6de0400101640159b8.pdf

16. Lohman T, Roche A, Martorell R. Anthropometric standarization reference manual. Champlaign, IL: Human Kinetics, 1988.

17. Habicht JP. Estandarización de métodos epidemiológicos cuantitativos sobre el terreno (Standardization of anthropometric methods in the field). PAHO Bull. 1974;76:375-84.

18. STATA versión 10.1. StataCorp. Stata Statistical Software: Release 12.1 College Station, TX: StataCorp LP. 2011.

19. Instituto Nacional de Estadísticas de Chile. VII Encuesta de Presupuestos Familiares. [citado 13/03/2015] Disponible en: http://www.ine.cl/epf/

20. Vereecken C, Pedersen TP, Ojala K, Krolner R, Dzielska A, Ahluwalia N, et al. Fruit and vegetable consumption trends among adolescents from 2002 to 2010 in 33 countries. Eur J Public Health 2015;25 Suppl 2:16-9.

21. Yngve $A$, Wolf $A$, Poortvliet $E$, et al. Fruit and vegetable intake in a sample of 11-year-old children in 9 European countries: the Pro Children Cross-sectional Survey. Ann Nutr Metab. 2005;49:236-45.

22. Diethelm K, Jankovic N, Moreno LA, et al. Food intake of European adolescents in the light of different foodbased dietary guidelines: results of the HELENA (Healthy Lifestyle in Europe by Nutrition in Adolescence) Study. Public Health Nutr. 2012;15:386-98.

23. Jimenez-Aguilar A, Gaona-Pineda EB, Mejia-Rodriguez F, Gomez-Acosta LM, Mendez-Gomez Humaran I, FloresAldana M. Consumption of fruits and vegetables and health status of Mexican children from the National Health and Nutrition Survey 2012. Salud Publica Mex. 2014;56 Suppl 2:s103-12.

24. Fonseca Z, Heredia A, Ocampo P, Forero $Y$, Sarmiento O, Alvarez $M$, et al. Encuesta nacional de la situación nutricional en Colombia 2010, Primera Ed, ICBF, PROFAMILIA, MSPS, INS, p. 513, 2011.

25. Olivares S, Bustos N, Lera L, Zelada E. Estado nutricional, consumo de alimentos y actividad física en escolares mujeres de diferente nivel socioeconómico de Santiago de Chile. Rev Méd Chil. 2007 ;135( 1 ): 71-8.

26. Kristjansdottir AG, De Bourdeaudhuij I, Klepp KI, Thorsdottir I. Children's and parents' perceptions of the determinants of children's fruit and vegetable intake in a lowintake population. Public Health Nutr 2009;12(8):1224-33.

27. Rakhshanderou S, Ramezankhani A, Mehrabi Y, Ghaffari M. Determinants of fruit and vegetable consumption among Tehranian adolescents: A qualitative research. J Res Med Sci. 2014;19(6):482-9.

28. Krolner R, Rasmussen M, Brug J, Klepp KI, Wind M, Due P. Determinants of fruit and vegetable consumption among children and adolescents: a review of the literature. Part II: qualitative studies. Int J Behav Nutr Phys Act. 2011;8 (1):112.

29. Johansson L, Solvoll K, Bjørneboe GE, Drevon CA. Underand overreporting of energy intake related to weight status and lifestyle in a nationwide sample. Am J Clin Nutr. 1998;68:266-74. 